Ann. Biol. anim. Bioch. Biophys., 1977, 17 (4), 607-613.

\title{
Etude in vivo chez le rat de la production et de l'utilisation de l'acide lactique. Rôle du tube digestif
}

\author{
par C. REMESY, C. DEMIGNÉ, Jocelyne AUFRERE
}

Station de Physiopathologie de la Nutrition, I.N.R.A.,

Theix, Saint Genes Champanelle, 63110 Beaumont.

Summary. In vivo studies of production and utilization of lactic acid in rat : role of the digestive tract.

Digestive production of lactate of both microbial (D and $L$ forms) and metabolic origin (glycolysis in intestinal cells) was studied in rat. With a cereal-based diet, there was high lactic acid concentration in the stomach, especially in the non-secretory area where the $\mathrm{pH}$ was approximately 5 (63.1 and $78.0 \mathrm{mM}$ of $\mathrm{D}$ and $\mathrm{L}$ lactate). In the caecum, the lactate concentration increased only when the caecal pH fell below 5 .

In fed animals, the small intestine produced lactate, partly taken up by the liver (10 to 15 p. 100 of the lactate in the portal vein) or by the peripheral tissues (iliac veinaorta). During fasting, digestive tract lactate production considerably decreased, while liver uptake (percentage) increased in relation to the increase in Cori cycle activity and gluconeogenesis.

\section{Introduction.}

Le métabolisme du lactate dans l'organisme est particulièrement complexe puisque de nombreux tissus peuvent, selon les circonstances, produire ou oxyder du lactate. Le lactate provenant de la glycolyse de certains tissus (hématies, muscles lisses, poumon etc...) peut correspondre à une utilisation différée du glucose vers d'autres tissus (cœur, rein, muscle rouge), ou à un recyclage du glucose vers la néoglucogenèse hépatique (cycle de Cori). Le turnover du lactate augmente chez l'animal nourri bien que l'activité du cycle de Cori soit alors minimum (Freminet et al. 1975, 1976). La production du lactate par le tube digestif a souvent été sous-estimée (Minaire, 1973), qu'elle soit due à la glycolyse par les entérocytes ou à l'absorption de lactate provenant des fermentations digestives.

Cet article se propose de préciser le rôle du tube digestif dans l'élévation de la production de lactate chez le rat nourri en comparant l'origine fermentaire et métabolique (glycolyse intestinale) du laciate. De plus nous décrirons les caractéristiques du métabolisme du lactate au niveau du foie et des membres inférieurs chez le rat nourri ef à jeun. 


\section{Matériel et méthodes.}

Animaux. Des rats Sherman mâles de 200 à $250 \mathrm{~g}$ ont été utilisés après anesthésie au Nembutal (40 mg/kg, s.c.).

Régimes. Les animaux recevaient à volonté les régimes suivants :

Régime « normal », aliment granulé Sanders (91260 Juvisy/Orge) à base de blé, maïs, tourteaux de soja et mélasses apportant en pourcentage : protéines (20), lipides (2), matières cellulosiques $(4,5)$, minéraux (7).

Régime semi-synthétique comprenant en pourcentage : caséine (13), amidon de froment (79), huile de maīs (2), minéraux (5), vitamines (1). Les mélanges vitaminiques et minéraux ont été établis par UAR (91360 Villemoisson/Orge).

Régime semi-synthétique enrichi en amidon cru de pomme de terre : entre 20 et $79 \mathrm{p}$. 100 selon le taux de remplacement de l'amidon de froment.

Prélèvement. Les prélèvements sont effectués après une période minimale de 10 jours d'adaptation au régime.

Contenus digestifs : des échantillons de 0,2 à $1 \mathrm{~g}$ de contenu sont placés dans des microtubes Eppendorf et immédiatement congelés à $-20^{\circ} \mathrm{C}$.

Le sang est ponctionné dans l'une des veines du fube digestif (gastrique, jéjunale, cæcale ou porte) ainsi que dans l'aorte abdominale, récolté sur héparine, centrifugé et le plasma conservé à $-20^{\circ} \mathrm{C}$.

Techniques biochimiques. Les contenus digestifs congelés sont centrifugés ( $2 \mathrm{mn}$ $16000 \mathrm{t} / \mathrm{mn}$ ) et 1 volume de surnageant est déprotéinisé par $\mathrm{HClO}_{4} 0,4 \mathrm{M}$ (49 v pour les contenus gastriques, $19 \mathrm{v}$ pour les autres contenus). Le plasma est déprotéinisé par $2 \vee$ d'HClO $0,6 \mathrm{M}$. L'acide lactique est dosé par action simultanée de la GPT et de la $D$ ou $L$ lacłate déshydrogénase en présence de NAD (Noll, 1974). Les acides gras volatils (AGV) ont été dosés dans les contenus digestifs selon les techniques précédemment décrites (Remesy, 1973 ; Remesy et Demigné, 1976). Le glucose ef l'alanine ont été déterminés par les méthodes enzymatiques de Slein (1965) et de Williamson (1974).

\section{Résultats et discussion.}

1. Répartition du lactate dans le tube digestif du rat.

Estomac (tabl. 1). - Avec le régime céréales + tourteaux, le $\mathrm{pH}$ des contenus digestifs est beaucoup plus élevé dans la zone non sécrétoire (5,0 contre 3,6 dans la zone sécrétoire). Les fermentations, acétique et lactique sont maximales dans la zone non sécrétoire. La présence d'acide D. lactique (35 p. 100 de l'acide lactique) indique l'origine fermentaire de ces produits. L'hétérogénéité du contenu gastrique est favorisée par la présence d'éléments peu solubles dans la ration, ainsi que par la coprophagie. Avec un régime semi-synthétique le contenu, plus liquide, ne permet pas l'établissement d'un gradient de $\mathrm{pH}$; les concentrations en acide lactique sont très faibles (forme L principalement, sans doute d'origine endogène). 
Intestin grêle (tabl. 1). - La teneur en L-lactate tend à augmenter légèrement dans les contenus du jéjunum et de l'iléon à l'inverse du D lactate; il existe sans doute un transfert de L-laciate d'origine endogène (Ducluzeau et al., 1966) depuis la muqueuse intestinale, qui est le siège d'une glycolyse intense (Hanson ef Parsons, 1976) vers le contenu digestif. Ce transfert rendrait compte du L-lactate mesuré dans les contenus d'intestin grêle avec un régime à 79 p. 100 d'amidon de froment puisqu'on ne retrouve pas de D-Lactate dans ce cas.

\section{TABLEAU 10}

Répartifion de l'acide lactique ( $L$ ef D) ef des acides gras volatils (AGV) dans l'estomac ef l'intestin grêle du rat nourri (prélèvements en fin de période absorptive). Cas d'un régime à base de céréales + tourteaux de soja. Les contenus gasłriques ont été étudiés dans 2 zones : estomac I (zone non sécrétoire transparente) et estomac II (zone sécrétoire opaque ef de couleur rougeâtre).

\begin{tabular}{|c|c|c|c|c|c|c|c|}
\hline & $\mathrm{pH}$ & $\begin{array}{c}\text { AG } V \text { totaux } \\
(\mathrm{mM})\end{array}$ & $\begin{array}{c}\text { Acide } \\
\text { acétique } \\
\text { (p. 100) }\end{array}$ & $\begin{array}{l}\text { Acide } \\
\text { L lactique } \\
(\mathrm{mM})\end{array}$ & $\left\{\begin{array}{c}\text { Acide } \\
D \text { lactique } \\
(\mathrm{mM})\end{array}\right.$ & $\begin{array}{c}\text { Rapport } \\
\text { L/D }\end{array}$ & $\begin{array}{l}\text { Matière sèche } \\
\text { (p. 100) }\end{array}$ \\
\hline $\begin{array}{l}\text { Estomac I. } \\
\text { Estomac II. } \\
\text { Duodénum } \\
\text { Jéjunum. . } \\
\text { lléon...... }\end{array}$ & $\begin{array}{l}5,00 \pm 0,09 \\
3,59 \pm 0,14 \\
6,18 \pm 0,13 \\
6,69 \pm 0,05 \\
6,80 \pm 0,10\end{array}$ & $\begin{array}{r}57,4 \pm 4,0 \\
14,9 \pm 1,7 \\
1,5 \pm 0,2 \\
6,1 \pm 0,8\end{array}$ & $\begin{array}{l}97,6 \\
93,3 \\
100 \\
\overline{100}\end{array}$ & $\begin{array}{r}78,0 \pm 5,6 \\
25,0 \pm 3,6 \\
7,1 \pm 0,9 \\
10,5 \pm 1,5 \\
16,6 \pm 2,6\end{array}$ & $\mid \begin{array}{r}63,1 \pm 4,5 \\
13,4 \pm 2,0 \\
3,3 \pm 0,6 \\
2,3 \pm 0,5 \\
1,5 \pm 0,2\end{array}$ & $\begin{array}{r}1,24 \\
1,87 \\
2,20 \\
5,23 \\
11,07\end{array}$ & $\begin{array}{c}45,4 \pm 0,8 \\
31,0 \pm 1,2 \\
- \\
20,2 \pm 0,9\end{array}$ \\
\hline
\end{tabular}

TABLEAU $1 b$

Cas d'un régime semi-synthétique à base d'amidon de froment comportant 13 p. 100 de caséine. Le contenu gastrique ne présentant pas d'hétérogénéité notable a été éfudié globalement. Les concentrations en AGV sont très faibles dans l'estomac et l'intestin grêle avec ce type de régime.

Les résultats sont les moyennes pour 8 animaux $\pm \sigma / \sqrt{n}$.

\begin{tabular}{|c|c|c|c|c|}
\hline & $\mathrm{pH}$ & $\begin{array}{l}\text { Acide } \\
\text { L lactique }\end{array}$ & $\begin{array}{c}\text { Acide } \\
\text { D lactique }\end{array}$ & Rapport \\
\hline $\begin{array}{l}\text { Estomac } \ldots \ldots \ldots \ldots \ldots \ldots \ldots \\
\text { Duodénum } \ldots \ldots \ldots \ldots \ldots \ldots \ldots \\
\text { Jéjunum } \ldots \ldots \ldots \ldots \ldots \ldots \ldots \ldots \\
\text { Iléon } \ldots \ldots \ldots \ldots \ldots \ldots \ldots \ldots\end{array}$ & $\begin{array}{l}4,14 \pm 0,25 \\
5,54 \pm 0,25 \\
6,88 \pm 0,03 \\
7,13 \pm 0,10\end{array}$ & $\begin{array}{l}5,20 \pm 0,80 \\
1,07 \pm 0,14 \\
2,44 \pm 0,30 \\
2,65 \pm 0,90\end{array}$ & $\begin{array}{c}107 \pm 0,14 \\
0 \\
0 \\
0\end{array}$ & $\begin{array}{l}4,86 \\
- \\
-\end{array}$ \\
\hline
\end{tabular}

Caecum. - Les conditions régnant dans le cæcum ( $\mathrm{pH}$, substrats, flore) peuvent varier considérablement selon le type de ration (tabl. 2) :

- Régimes semi-synthétiques. Le cæcum est très peu développé, la présence d'azote endogène sans celle de glucides fermentescibles conduit à des fermentations de $\mathrm{pH}$ voisin de 7. On ne détecte alors pas d'acide lactique, mais seulement des AGV.

- Régimes normaux, apportant des quantités modérées de subsirats fermentescibles : céréales entières, tourteaux. Le poids du cæcum augmente, le $\mathrm{pH}$ est voisin de 
6 et les AGV présents en concentrations élevées, par contre l'acide lactique est inférieur à $8 \mathrm{mM}$ (forme $L$ surtout).

- Régimes riches en substrats fermentescibles (amidon cru de pomme de terre à des taux compris entre 30 et 50 p. 100). Ces régimes conduisent à un développement considérable du cæcum et à une baisse du $\mathrm{pH}$ : jusque vers $\mathrm{pH} 5$ les fermentations à AGV dominent, mais l'état d'oxydoréduction du milieu favorise la production de butyrate et de lacłate aux dépens de celle de propionate. Lorsque le $\mathrm{pH}$ descend vers 4,5 le poids du cæcum devient considérable (15 g) et la flore produit presque uniquement de l'acide lactique et acétique comme dans l'estomac. Dans ce cas la matière sèche des fèces esł faible, l'adaptation à ces régimes, même progressive donne des résultats inconstants (parfois pertes de poids).

\section{TABLEAU 2}

Comparaison de la concentration d'ocide $D, L$ lactique ef d'AGV dans le cæcum de rats nourris avec des régimes apportant des quantités croissantes de glucides fermentescibles au niveau du cæcum.

Les résultats sont les moyennes $\pm \sigma / \sqrt{\mathrm{n}}$ pour 10 animaux

\begin{tabular}{|c|c|c|c|c|c|c|c|c|}
\hline Régime & $\begin{array}{l}\text { Poids du } \\
\text { cæcum (g) } \\
\text { (rats de } \\
200 \mathrm{~g} \text { ) }\end{array}$ & $\mathrm{pH}$ & $\begin{array}{c}\text { AGV } \\
\text { totaux } \\
(\mathrm{mM})\end{array}$ & $\begin{array}{c}\text { Acide } \\
\text { acétique } \\
\text { (p. 100) }\end{array}$ & $\begin{array}{l}\text { Acide } \\
\text { propionique } \\
\text { (p. 100) }\end{array}$ & $\begin{array}{c}\text { Acide } \\
\text { butyrique } \\
\text { (p. 100) }\end{array}$ & $\mid \begin{array}{c}\text { Acide } \\
\text { L-lactique } \\
(\mathrm{mM})\end{array}$ & $\begin{array}{c}\text { Acide } \\
\text { D-lactique } \\
(\mathrm{mM})\end{array}$ \\
\hline $\begin{array}{l}\text { Amidon Froment } \\
\text { Céréales }+ \text { Tour- }\end{array}$ & $2,5 \pm 0,1$ & $6,98 \pm 0,04$ & $4118,3 \pm 11,4$ & 68,3 & 25,1 & 6,6 & 0 & 0 \\
\hline $\begin{array}{l}\text { teaux } \ldots \ldots \ldots \\
\text { Amidon cru de } \\
\text { pomme de terre } \\
\text { (35 p. 100).... }\end{array}$ & $4,3 \pm 0,3$ & $5,81 \pm 0,11$ & $146,6 \pm$ & 59,1 & 25,3 & 15,6 & $\begin{array}{l}4,3 \pm 0,9 \\
9,6 \pm 1,5\end{array}$ & $\begin{array}{l}1,9 \pm 0,4 \\
8,1 \pm 1,0\end{array}$ \\
\hline $\begin{array}{l}\text { Amidon cru de } \\
\text { pomme de terre* } \\
(79 \text { p. 100) .... }\end{array}$ & $15,3 \pm 0,5$ & $4,65 \pm 0,10$ & $74,4 \pm 2,6$ & 69,1 & 5,9 & 0,3 & 70,4 & $\pm 10,0$ \\
\hline
\end{tabular}

* Pour ce régime, des rats Wistar ont été utilisés, avec comme apport protéique de la farine de hareng

2. Comparaison du métabolisme du jéjunum et du caecum.

Le jéjunum est le siège d'une forte absorption de glucose avec la production de lactate et d'alanine alors que le cæcum consomme du glucose et libère peu de lactate (tabl. 3). Les différences artérioveineuses dans la veine porte correspondent à celles au niveau du jéjunum, bien que plus faibles du fait de la dilution par le sang provenant des autres parties du tube digestif. Les régimes semi-synthétiques ne favorisent pas la production de lactate par la flore; on enregistre néanmoins d'importantes différences veine porte-artère en $L$ lactate durant la phase absorptive, dues à la glycolyse de l'intestin (tabl. 4).

La transformation des glucides par la paroi de l'intestin grêle assure la majeure partie de la production de lactate par le tube digestif du rat. Celle-ci semble d'autant plus forte que l'absorption du glucose est élevée (veine porte-artère ; tabl. 3 et 4). 
Cependant le pourcentage de transformation en lactate du glucose absorbé est d'autant plus important que son absorption est faible. A jeun, la production intestinale de lactate représente 22 p. 100 du glucose capté, valeur proche de celle des territoires drainés par la veine iliaque (17 p. 100). Si les cellules de la muqueuse assurent une part de la production intestinale du lactate (Sherratt, 1968), on conçoit l'utilisation privilégiée du glucose de la lumière ; toutefois des auteurs comme Leese et Bronk (1975) attri-

\section{TABLEAU 3}

Bilans artérioveineux en glucose, lacfote et alanine à différents niveaux du tube digestif. Les rats nourris (régime céréales plus tourteaux) ont été abattus en fin de la période absorptive. Les résultats sont les moyennes $\pm \sigma / \sqrt{n}$ pour 10 animaux.

\begin{tabular}{|c|c|c|c|}
\hline & $\begin{array}{c}\text { Glucose } \\
(\mathrm{mM} M)\end{array}$ & $\begin{array}{c}\text { Lactate } \\
(\mathrm{mM})\end{array}$ & $\begin{array}{c}\text { Alanine } \\
(\mathrm{m} M)\end{array}$ \\
\hline 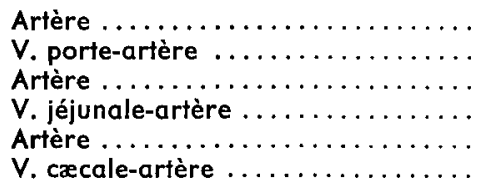 & $\begin{array}{r}7,00 \pm 0,10 \\
+1,10 \pm 0,15 \\
7,20 \pm 0,22 \\
+1,73 \pm 0,26 \\
7,50 \pm 0,30 \\
-0,40 \pm 0,10\end{array}$ & $\begin{array}{r}2,00 \pm 0,21 \\
+0,52 \pm 0,11 \\
1,87 \pm 0,05 \\
+1,13 \pm 0,10 \\
1,97 \pm 0,10 \\
+0,16 \pm 0,05\end{array}$ & $\begin{array}{r}0,39 \pm 0,03 \\
+0,33 \pm 0,01 \\
0,34 \pm 0,01 \\
+0,49 \pm 0,04 \\
0,36 \pm 0,07 \\
+0,09 \pm 0,01\end{array}$ \\
\hline
\end{tabular}

buent à la zone musculaire un rôle prépondérant. Les mécanismes de ceffe production de lactate restent obscurs : Jodal ef Lundgren (1970) invoquent l'oxygénation insuffisante de certaines zones due à une baisse du nombre d'hématies dans le sang qui irrigue les villosités. Le rapport AMP + ADP/ATP élevé, observé par Lemhoff et al. (1970) peut expliquer une glycolyse aérobie intense dans l'intestin ; toutefois Lamers et Hülsmann (1972), s'appuyant sur l'existence d'un effet Pasteur (controversé), estiment que le lactate n'est produit que dans des conditions d'anaérobiose. Le lactate repré-

\section{TABLEAU 4}

Comparaison du métabolisme du lactote et du glucose dans différents territoires de l'organisme du rat nourri (régime amidon de froment +13 p. 100 de caséine) ou à jeûn $(48 \mathrm{~h})$. Les valeurs positives indiquent une production et les valeurs négatives une captation. Les résultats sont les moyennes $\pm \sigma / \sqrt{n}$ pour 10 animaux.

\begin{tabular}{|c|c|c|c|c|}
\hline & \multicolumn{2}{|c|}{ Nourri } & \multicolumn{2}{|c|}{ Jeûne (48 h) } \\
\hline & $\begin{array}{c}\text { Glucose } \\
(\mathrm{mM})\end{array}$ & $\begin{array}{l}\text { Lactate } \\
(\mathrm{mM})\end{array}$ & $\begin{array}{c}\text { Glucose } \\
(\mathrm{mM})\end{array}$ & $\begin{array}{c}\text { Lactate } \\
(\mathrm{mM})\end{array}$ \\
\hline $\begin{array}{l}\text { Artère } \ldots \ldots \ldots \ldots \ldots \ldots \\
\text { V. porte-artère } \ldots \ldots \ldots \ldots \ldots \\
\text { V. iliaque-artère } \ldots \ldots \ldots \ldots \\
\text { V. hépatique-v. porte } \ldots \ldots \ldots\end{array}$ & $\begin{array}{r}8,20 \pm 0,20 \\
+5,05 \pm 0,33 \\
-0,80 \pm 0,14 \\
-3,40 \pm 0,40\end{array}$ & $\begin{array}{r}2,34 \pm 0,10 \\
+0,88 \pm 0,07 \\
-0,40 \pm 0,06 \\
-0,48 \pm 0,12\end{array}$ & $\begin{array}{r}6,75 \pm 0,19 \\
-0,93 \pm 0,14 \\
-0,52 \pm 0,11 \\
+2,15 \pm 0,15\end{array}$ & $\begin{array}{r}1,03 \pm 0,04 \\
+0,38 \pm 0,02 \\
+0,18 \pm 0,07 \\
-0,98 \pm 0,07\end{array}$ \\
\hline
\end{tabular}


senterait également l'aboutissement du métabolisme de divers acides organiques (Worbe, Mottaz et Sadeghi, 1969) dans le jéjunum. De même, dans le cæcum de lapin Marty et Carles (1973) montrent que le glucose capté ne peut assurer la synthèse de tout le lactate produit (participation de divers acides organiques) ; toutefois selon nos résultats la consommation de glucose explique la plus grande partie du lactate libéré par la paroi cæcale. L'élévation du turnover du lactate chez l'animal nourri n'a pas reçu d'explication très satisfaisante (Freminet ef al., 1976). La glycolyse intestinale et parfois l'absorption de lactate fermentaire rendent compte sans doute d'une grande partie de ce phénomène.

\section{Comparaison de l'utilisation hépatique ef périphérique du lactate (tabl. 4).}

Nos données apportent des éléments nouveaux en soulignant :

a) au niveau splanchnique l'existence d'une libération intestinale de lactate suivie d'une captation hépatique (vers l'oxydation ef la lipogenèse), chez le rat nourri ;

b) la faible libération intestinale de lactate à jeun et la captation hépatique accrue (vers la néoglucogenèse) malgré la baisse du lactate dans le sang porte ;

c) les fluctuations du métabolisme périphérique, orienté chez le rat nourri, vers l'utilisation du lactate lorsque les glycémies et les lactacidémies sont élevées et les acides gras libres (AGL) faibles (Dunn et Critz, 1975) ou à jeun, vers la production de lactate (Mc Donald ef al., 1976) en raison de l'inhibition de la pyruvate déshydrogénase exercée par les AGL et les corps cétoniques (Berger et al., 1976). Les échanges de lactate se conforment alors aux lois du cycle de Cori.

Commission CNERNA Digestion-Absorption, Tours, 13 novembre 1976.

\section{Références}

BERGER M., HAGG S. A., GOODMAN M. M., RUDERMAN N. B., 1976. Glucose metabolism in perfused skeletal muscle. Effects of starvation diabetes, fatty acids, acetoacetate, insuline and exercice on glucose uplake and disposition. Biochem. J., 158, 191-202.

DUCLUZEAU R., RAIBAUD P., SACQUET E., MOCQUOT G., 1966. Mise en évidence in vivo d'une production d'acide lactique dans le tube digestif de rats axeniques à monoflore de lactobacilles. C. R. Acad. Sc. Paris., 262, 321-324.

DUNN R. B., CRITZ J. B., 1975. Uptake of lactate by dog skeletal muscle in vivo and the effect of free fatty acids. Am. J. Physiol., 229, 255-259.

FREMINET A., LECLERC L., GENTIL M., POYART C., 1975. Effect of fasting on the rates of lactate turnover and oxidation in rats. Febs Letf., 60, 431-434.

FREMINET A., POYART C., LECLERC L., GENTIL M., 1976. Effect of fasting on the Cori cycle in rats. Febs Lett., 66, 328-331.

HANSON P. J., PARSONS D. S., 1976. The utilization of glucose and production of lactate by in vitro preparations of rat small intestine : effects of vascular perfusion. J. Physiol., 255, 775-795.

IEMHOFF W. G. J., VAN DEN BERG J. W. O., DE PIJPER A. M., HÜLSMANN W. C., 1970. Metabolic aspects of isolated cells from rat small intestinal epithelium. Biochim. Biophys. Acta, 215, 229-241. 
JODAL. M., LUNDGREN O., 1970. Plasma skimming in the intestinal tract. Acto physiol. scand., 80, 50-60.

LAMERS J. M. J., HÜLSMANN W. C., 1972. Pasteur effect in the in vitro vascularly perfused rat liver. Biochim. Biophys. Acto, 275, 491-495.

LEESE H. J., BRONK J. R., 1975. Lactate formation by rat small intestine in vitro. Biochim. Biophys. Acta, 404, 40-48.

Mc DONALD M., NEUfELDT M., PARK M. B., BERGER M., RUDERMANN N. B., 1976, Alanine metabolism and gluconeogenesis in the rat. Am. J. Physiol., 231, 619-626.

MARTY J., CARLES J., 1973. Absorption des acides carboxyliques par la paroi cæcale au lapin. Ann. Biol. anim. Bioch. biophys., 13, 453-464.

MINAIRE Y., 1973. Origine et destinée du lactate plasmatique. J. Physiol., Paris, 66, 229-257.

NOLL F., 1974. Lactic acid determination with LDH, GPT and NAD, 1475-1479. In Bergmeyer H. U., Methods of enzymatic analysis (2nd Ed), Acad. Press, New York, London.

REMESY C., 1973. Contribution à l'étude de la production et du métabolisme des acides gras volatils chez le rat. Thèse $3^{\mathbf{e}}$ cycle, Clermont-Ferrand.

REMESY C., DEMIGNÉ C., 1976. Partition and absorption of volatile fatty acids in the alimentary canal of the rat. Ann. Rech. vétér., 7, 39-55.

SHERRATT S. A., 1968. The metabolism of small intestine. Oxygen uptake and L. Lactate production along the length of the small intestine of the rat and guinea pig. Comp. Biochem. physiol., 24, 745-761.

SLEIN M. W., 1965. In BERGEMEYER H. U. Methods of enzymatic analysis. Acad. Press, New York, London 117-123.

WILLIAMSON D. H., 1974. L. alanine determination with alanine deshydrogenase, 1678-1682. In BERGEMYER H. U. Methods of enzymatic analysis (2nd ed.), Acad. Press, New York, London.

WORBE J. F., MOTTAZ P., SADEGHI R., 1969. Influence de l'acide succinique et de l'acide $\alpha$-cétoglutarique sur l'activité métabolique de l'intestin grêle du rat. C. R. Soc. Biol., 163, 431-436. 